in Übereinstimmung mit den jüngsten Messungen ${ }^{35,36}$, von denen die genauesten Werte zwischen 1,695 und 1,712 mit einem Fehler von 0,005 bis 0,008 lieferten $^{36}$.

${ }^{35}$ H. T. Motz, Phys. Rev. 85, 501 [1952]; E. K. Hyde u. G. D. O'Kelley, ebd. 82, 944 [1951]; H. M. Agnew, ebd. 77, 655 [1950]; L. M. Langer u. H. C. Price jr., ebd. 76, 641 [1949]; W. C. Peacock, J. W. Jones u. R. T. Overman, Plutonium Project Report Mon N-432, 56 [1947] (zitiert aus ${ }^{2}$ ); C. M. Witcher,
Herrn Prof. J. H. D. Jensen danken wir für belehrende Diskussionen über das Kernschalenmodell.

Für diese Untersuchung wurden Apparate der Deutschen Forschungsgemeinschaft mitbenutzt.

Phys. Rev. 60, 32 [1941]; J. L. Lawson, ebd. 56, 131 [1939].

${ }^{36}$ E. N. Jensen, R. T. Nichols, J. Clement u. A. Pohm, Phys. Rev. 85, 112 [1952]; R. K. Sheline, R. B. Holtzman u. C.-Y. Fan, ebd. 83, 919 [1951]; S. D. Warshaw, J. J. L. Chen u. G. L. Appleton, ebd. 80, 288 [1950]; K. Sieg bahn, ebd. 70, 127 [1946].

\title{
Zur Kinetik des konvektiven Wärmeüberganges und der Strömungsreibung an spiegelnden Oberflächen
}

\author{
Von EUgen SäNGER* \\ (Z. Naturforschg. 9a, 410-418 [1954]; eingegangen am 30. Januar 1954)
}

\begin{abstract}
Eine der ernstesten Schwierigkeiten des Fluges mit hohen Überschall-Mach-Zahlen besteht bekanntlich in der Reibungserwärmung der schnellen Gase in den Strömungsgrenzschichten und in dem damit verbundenen hohen konvektiven Wärmeübergang an die vom Fahrtwind oder von den Antriebsstrahlen berührten festen Oberflächen fliegender Körper.

Es werden die gaskinetischen Bedingungen studiert, die durch Anwendung spiegelnd glatter Wände - d. h. solcher mit Rauhigkeiten die vergleichbar mit den Materiewellenlängen der auftreffenden Korpuskularstrahlen sind — zu einer erheblichen Herabsetzung von Wärmeübergang und Reibung führen könnten.

Die Ansprüche an die Glätte solcher Spiegelwände erweisen sich als sehr erheblich und als unabhängig von der Strömungsgeschwindigkeit. Atmosphärische Luft zeigt überwiegende Spiegelreflexion erst bei Wandrauhigkeiten zwischen $10^{-9}$ und $10^{-8} \mathrm{~cm}$, dasselbe gilt für das atomare Wasserstoffgas bzw. Protonengas der Plasmen von etwa $10000^{\circ} \mathrm{K}$, während das Elektronengas solcher Plasmen schon bei Rauhigkeiten zwischen $10^{-8}$ und $10^{-7} \mathrm{~cm}$ überwiegend spiegelnd reflektiert wird. Vergleichsweise beginnt sichtbares Licht bei Rauhigkeiten von $10^{-4} \mathrm{~cm}$ gespiegelt zu werden.

Endlich werden geschlossene Formeln gegeben für die Beiträge zu Wärmeübergang und Reibung der drei kinetischen Grenzflächenvorgänge:

1. Aufprall des dann an der Wand haftenden Partikelanteils (positiver Wärmeübergang und Reibung);

2. Diffuse Wiederauslösung des an der Wand haftenden Partikelanteils (negativer Wärmeübergang, kein Reibungsbeitrag);

3. Elastische Reflexion des spiegelnd zurückgeworfenen Partikelanteils (positiver oder negativer Wärmeübergang, kein Reibungsbeitrag).
\end{abstract}

$\mathrm{D}^{-}$ üblichen Grenzschichttheorie der Strömungsreibung an einer Wand und des konvektiven Wärmeüberganges an die Wand liegt die Annahme zugrunde, daß die Grenzschicht an der Wand „,haftet", oder gaskinetisch gesprochen, daß die aufprallenden Gasmoleküle nicht spiegelnd, sondern diffus reflektiert werden.

Aus Molekularstrahlversuchen ist bekannt, daß dies im allgemeinen auch zutrifft, daß aber in Analogie zu den Photonenstrahlen auch Atom- und Molekularstrahlen dann spiegelnd an einer Wand reflektiert werden, wenn die Höhe $\xi$ der Uneben-

* Courcelle-sur-Yvette (S.- \& -O.), France. heiten der Wand, auf den Strahl projeziert, kleiner wird, als die de Broglie-Wellenlänge $\lambda=h / m w$ des Strahles.

Darin ist $h$ das Plancksche Wirkungsquantum, $m$ die Partikelmasse und $w$ ihre Auftreffgeschwindigkeit.

Bezeichnet man noch mit $\alpha$ den wirklichen und mit $\alpha_{\mathrm{k}}$ den kritischen Auftreffwinkel, so gilt also für spiegelnde Reflexion:

$$
\sin \alpha \leqq \sin \alpha_{\mathbf{k}}=\lambda / \xi=h / m w \xi
$$

Diese Beziehung entspricht etwa der Braggschen Beziehung der Röntgenoptik und gilt daher auch, wenn die de Broglie-Wellenlänge der Gaspartikel 
im Gebiet der Röntgenwellenlängen liegt, was beispielsweise für Luft im gesamten technisch interessierenden Flug-Mach-Zahlbereich von 0 bis 40 zutrifft.

Der Grenzwinkel $\alpha_{k}$ wird gleich $\pi / 2, d$. h. es werden alle Gaspartikel spiegelnd reflektiert, welches auch ihr Auftreffwinkel sei, wenn die Molekulargeschwindigkeit

$$
w \leqq h / m \xi,
$$

also die Rauhigkeitshöhe $\xi$ kleiner als die Wellenlänge $\lambda$ wird, ein bekanntes, allgemeines Grundgesetz spiegelnder Reflexion.

Für die Rauhigkeit von Kristallspaltflächen oder Oberflächenfilmen normaler Zimmertemperatur von $\xi \sim 1 \AA$ trifft dies daher beispielsweise zu:

bei Molekulargeschwindigkeiten atmosphärischer

Luft unter etwa $w \leqq 138 \mathrm{~m} / \mathrm{sec}$,

bei den Partikelgeschwindigkeiten atomaren Was-

serstoffes unter etwa $w \leqq 4000 \mathrm{~m} / \mathrm{sec}$,

bei Elektronengeschwindigkeiten unter etwa

$w \leqq 7,290000 \mathrm{~m} / \mathrm{sec}$.

Für die Rauhigkeit geschliffener, technischer Flächen von $\xi \sim 10^{4} \AA$, die dem Auge in der senkrechten Aufsicht eben schon spiegelnd erscheinen, sind diese Grenzgeschwindigkeiten um vier Zehnerpotenzen kleiner, also für die erwähnten Beispiele

$$
0,0138 \mathrm{~m} / \mathrm{sec} ; 0,4 \mathrm{~m} / \mathrm{sec} \text { und } 729 \mathrm{~m} / \mathrm{sec} \text {. }
$$

Alle langsameren Partikel werden spiegelnd reflektiert.

\begin{tabular}{|l|r|c|}
\hline & $c_{1}[\mathrm{~m} / \mathrm{sec}]$ & $\lambda[\AA]$ \\
\hline $\begin{array}{l}\text { Luft von Stratosphären- } \\
\text { temperatur }\end{array}$ & 353 & 0,39 \\
\hline $\begin{array}{l}\text { atomarer Wasserstoff- oder } \\
\text { Protonengas von } 10000^{\circ} \mathrm{K}\end{array}$ & 12900 & 0,31 \\
\hline $\begin{array}{l}\text { Elektronengas von } \\
10000^{\circ} \mathrm{K}\end{array}$ & 550000 & 13,23 \\
\hline
\end{tabular}

Tab. 1. Häufigste oder wahrscheinlichste Partikelgeschwindigkeit

$$
c_{1}=\sqrt{2 k T / m}=\sqrt{2 g R T}
$$

und die entsprechenden Wellenlängen.

Diese in Tab. 1 erwähnten Gase müßten daher von zimmerwarmen Kristallspaltflächen schon zu

1 E. Sänger, Deutsche Luftfahrtforschung, F. B. 972, Berlin 1938. - Douglas Aircraft Corp., Transl. F. R. 369, Santa Monica 1946. - NACA, T. M. 1270, Washington DC 1950. einem gewissen Bruchteil spiegelnd reflektiert werden.

\section{Ruhendes Gas}

Der zunächst zu untersuchende Fall, daß die der Wand unmittelbar benachbarten Gasmassen keine Systemgeschwindigkeit parallel zur Wand besitzen, ist praktisch nicht nur dann gegeben, wenn die gesamte Gasmasse zur Wand in Ruhe ist, sondern in sehr guter Annäherung auch bei mit beliebig hohen Mach-Zahlen strömenden Gasen, die in Wandnähe eine Strömungsgrenzschicht ausbilden, bei denen also die freie Weglänge der Partikel oder der spiegelnd reflektierende Partikelanteil nicht zu hoch sind.

In diesen letzteren Fällen muß natürlich nicht die Temperatur der freien Strömung, sondern die wandnahe Grenzschichttemperatur den Rechnungen zugrunde gelegt werden, deren Höhe allerdings von vornherein meist unbekannt ist.

Bezeichnen wir mit $c$ die nach allen Richtungen des Raumes zielenden, thermischen Schwirrgeschwindigkeiten der Gaspartikel, so gruppieren sich diese nach der Maxwell-Boltzmann-Verteilung.

$$
\frac{\mathrm{d} \varrho}{\varrho}=\frac{4}{\sqrt{\pi}} \frac{c^{2}}{c_{1}{ }^{3}} e^{-c^{2} / c_{1}{ }^{2}} \mathrm{~d} c
$$

um die häufigste Geschwindigkeit $c_{1}$, wobei d $\varrho / \varrho$ der relative Massenanteil je Volumeinheit ist, dessen Geschwindigkeitsgröße zwischen $c$ und $c+\mathrm{d} c$ liegt. Wir greifen in Abb. 1 diesen Massenanteil d $\varrho$ fast homogener Schwirrgeschwindigkeit $c$ heraus. Beachten wir, daß d $\varrho \mathrm{d} F$ $4 c^{2} \pi$ die durch das Oberflächenelement $\mathrm{d} F=2 c^{2} \pi$ $\cos \alpha \mathrm{d} \alpha$ der gezeichneten

Geschwindigkeitskugel gehendePartikelmasse ist, so ergibt sich die sekundlich auf die Flächenein-

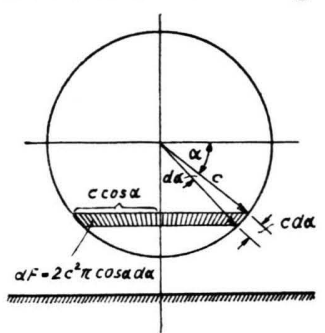

Abb. 1. Geometrie des Aufpralles der Partikel ruhenden Gases auf eine Wand. heit der Platte aufprallende Partikelmasse zu dem bekannten Wert ${ }^{1,2}$

$$
\mathrm{d} M=\frac{\mathrm{d} \varrho}{4 c^{2} \pi} \int_{\alpha=0}^{\pi / 2} c \sin \alpha \mathrm{d} F=\frac{c \mathrm{~d} \varrho}{4} .
$$

${ }^{2}$ E. Sänger, Schweizer Archiv f. angew. Wissenschaft u. Technik, 16, 43, Solothurn 1950. 
Davon wird also jener Anteil spiegelnd reflektiert werden, für den $\alpha<\alpha_{\mathrm{k}}$ ist:

$$
\begin{gathered}
\mathrm{d} M_{1}=\frac{\mathrm{d} \varrho}{4 c^{2} \pi} \int_{\alpha=0}^{\operatorname{arc} \sin h / m c \xi} c \sin \alpha \mathrm{d} F=\frac{c \mathrm{~d} \varrho}{4}\left(\frac{h}{m c \xi}\right)^{2} \\
=\frac{c \mathrm{~d} \varrho}{4} \sin ^{2} \alpha_{\mathrm{k}},
\end{gathered}
$$

soweit $\lambda / \xi=h / m c \xi<1$, andernfalls wird die Gesamtmasse $\mathrm{d} M$ spiegelnd reflektiert.

Es ist von gewisser praktischer Bedeutung, daß infolge der Geometrie des diffusen Aufpralles der spiegelnd reflektierende Anteil nicht einfach proportional $(h / m c \xi)$ ist, sondern proportional dem Quadrat dieses Winkelsinus, wodurch sich eine gegebene Verminderung der Rauhigkeit $\xi$ quadratisch auswirkt.

Bezieht man mit Hilfe der Maxwell-BoltzmannVerteilung alle Partikel mit den verschiedenen möglichen Geschwindigkeiten $c$ zwischen null und unendlich in die Rechnung ein, so wird die Gesamt. masse $M$ der sekundlich auf die Wandflächeneinheit aufprallenden Partikel, wie bekannt

$M=\int_{c=0}^{\infty} \frac{c \mathrm{~d} \varrho}{4}=\frac{\varrho}{\sqrt{\pi}} \int_{c=0}^{\infty} \frac{c^{3}}{c_{1}{ }^{3}} e^{-c^{2} / c_{1}{ }^{2}} \mathrm{~d} c=\frac{\varrho c_{1}}{2 \sqrt{\pi}}$

und die Gesamtmasse $M_{1}$ der mit kleinerem als dem kritischen Auftreffwinkel $\alpha_{\mathrm{k}}$ aufprallenden, also spiegelnd reflektierenden Partikel

$$
\begin{aligned}
M_{1} & =\int_{c=0}^{h / m \xi} \frac{c \mathrm{~d} \varrho}{4}+\int_{c=h / m \xi}^{\infty} \frac{c \mathrm{~d} \varrho}{4}\left(\frac{h}{m c \xi}\right)^{2} \\
& =\frac{\varrho c_{1}}{\sqrt{\pi}}\left[\int_{x=0}^{h / m c_{1} \xi} x^{3} e^{-x^{2}} \mathrm{~d} x+\left(\frac{h}{m c_{1} \xi}\right)^{2} \int_{x=h / m c_{1} \xi}^{\infty} x e^{-x^{2}} \mathrm{~d} x\right] \\
& =\frac{\varrho c_{1}}{2 \sqrt{\pi}}\left[1-e^{-\left(h / m c_{1} \xi\right)^{2}}\right] .
\end{aligned}
$$

Daher wird der spiegelnd reflektierende Bruchteil aller auftreffenden Partikel:

$$
\zeta=M_{1} / M=1-e^{-\left(h / m c_{1} \xi\right)^{2}} .
$$

Für sehr kleine $h / m c_{1} \xi$ wird nach Reihenentwicklung:

$$
\zeta \sim\left(h / m c_{1} \xi\right)^{2}=(\lambda / \xi)^{2} .
$$

Dieser Bruchteil ist also lediglich durch den auf die häufigste Partikelgeschwindigkeit $c_{1}$ bezogenen Wert $h / m c_{1} \xi$ bestimmt, der grundsätzlich auch gröBer als eins werden kann, also nicht mehr als Winkelsinus zu interpretieren ist. Erst für $h / m c_{1} \xi=0$ wird auch $\zeta$ gleich null, also streng genommen nie- mals. Daß hier auch $h / m c_{1} \xi>1$ werden kann, rührt daher, daß auch in diesem Fall noch Partikel mit $h / m c \xi<1$ enthalten sind, die diffus reflektiert werden. Erst mit $h / m c_{1} \xi \rightarrow \infty$ wird der diffus reflektierende Anteil verschwinden und daher $\zeta$ gegen eins gehen, wie Gl. (9) richtig wiedergibt.

Abb. 2 zeigt nach Gl. (9) den Bruchteil $\zeta$ spiegelnd reflektierender Partikel und zwar für Luftmoleküle, Wasserstoffatome, Elektronen und Photonen sichtbaren Lichtes, in Abhängigkeit von der Wandrauhigkeit $\xi$ und für verschiedene Gastemperaturen $T$.

Luft von Stratosphärentemperatur $T=216,5^{\circ} \mathrm{K}$ zeigt überwiegende Anteile spiegelnder Reflexion erst bei Rauhigkeiten zwischen $10^{-8}$ und $10^{-9} \mathrm{~cm}$, wie sie bei stark unterkühlten, idealgeordneten Flächen von Einkristallen denkbar sind, etwa bei der Temperatur des flüssigen Wasserstoffes, wobei die Freiheit dieser Oberflächen von adsorbierten Gasschichten und sonstigen Verunreinigungen eine wesentliche Rolle zu spielen scheint, die durch sehr niedrige Wandtemperaturen ungünstig beeinflußt wird.

Bei niedrigeren Lufttemperaturen steigt der Anteil, bei höheren Lufttemperaturen, wie sie gegebenenfalls in den Grenzschichten der Flugzeugoberflächen bei sehr hohen Flug-Mach-Zahlen herrschen, sinkt der Anteil spiegelnd reflektierender Luftmoleküle recht wesentlich ab.

Bei den heute üblichen Oberflächenrauhigkeiten fliegender Körper oder von Ofen- und Düsen-Wänden der Triebwerke (über $10^{-4} \mathrm{~cm}$ ) ist der Bruchteil spiegelnder Reflexion zwar nicht null, aber praktisch völlig vernachlässigbar und die übliche Annahme des Haftens der Strömungsgrenzschicht an der Oberfläche ist durchaus gerechtfertigt.

Während also der erste Schritt einer wesentlichen Herabsetzung der aerodynamischen Strömungsreibung und des Wärmeüberganges durch Erzwingung laminarer Grenzschichten ein Problem makroskopischer Formgebung der Wände (,Laminarprofile“) war, wird der weitere Schritt, durch Ausnützung spiegelnder Molekularreflexion zu geringerer Reibung und kleinerem Wärmeübergang zu kommen, ein sehr schwieriges Problem submikroskopischer Formgebung der Wandoberflächen, also überwiegend technologischer Natur. Entscheidende Fortschritte sind nur zu erwarten, wenn die Wandrauhigkeiten wesentlich unter die Größenordnung atomarer Rauhigkeiten (unter $10^{-8} \mathrm{~cm}$ ) gesenkt werden können. 
Etwas günstiger wird das Bild für leichtere Gase, da der kritische Parameter $h / m c_{1} \xi=h / \xi \sqrt{2 k T m}$ mit sinkender Partikelmasse wächst, wenn dieser Einfluß nicht durch erhöhte Gastemperaturen ausgeglichen wird.

Atomares Wasserstoffgas oder Protonengas von $10000{ }^{\circ} \mathrm{K}$, wie es etwa für thermische Atomraketen

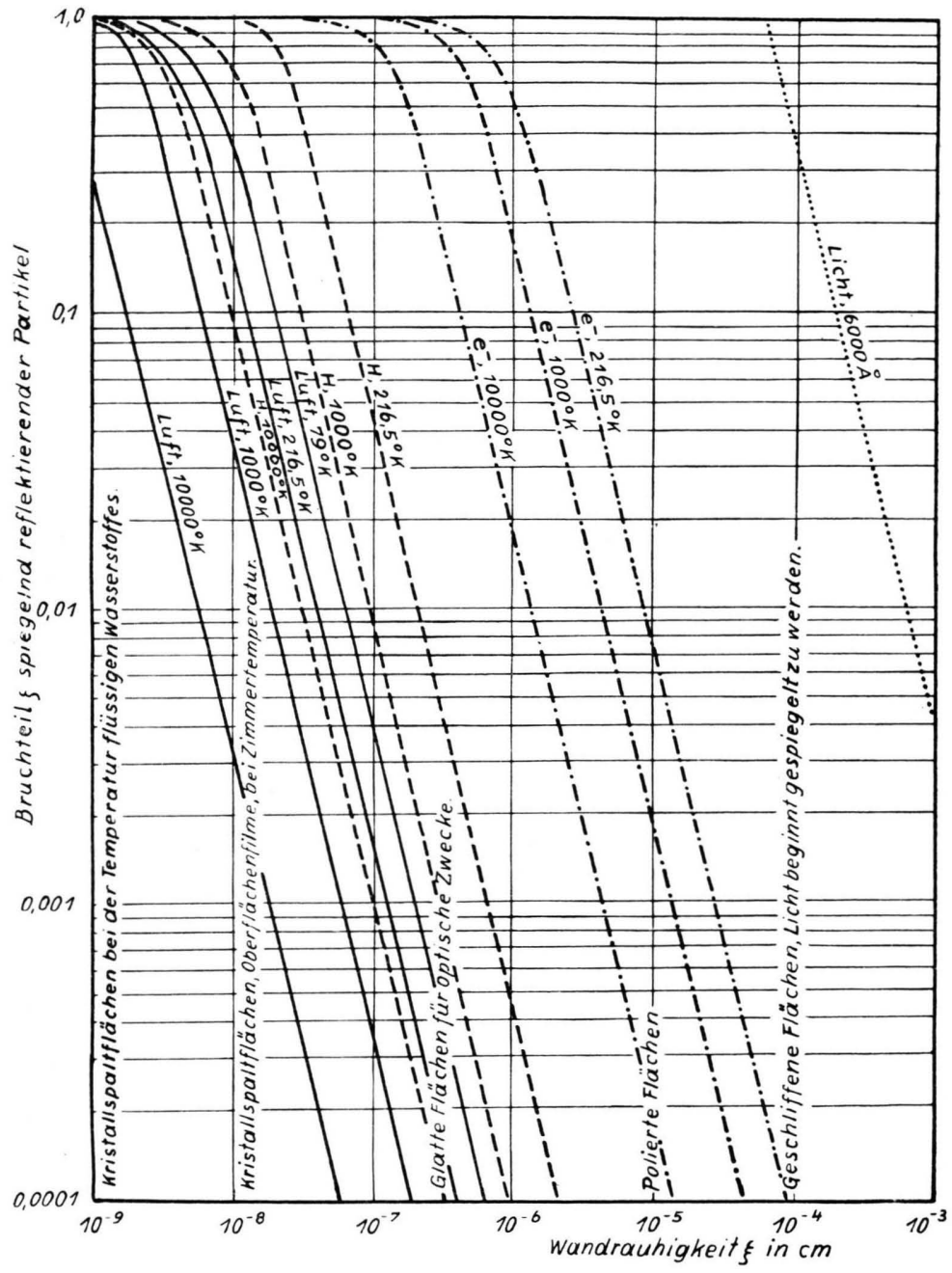

Abb. 2. Der Bruchteil $\zeta$ spiegelnd reflektierender Gaspartikel in Abhängigkeit von der Wandrauhigkeit $\xi$ und der Gastemperatur. (Luft; - - - atomarer Wasserstoff, Protonengas; - - - . - Elektronengas $\cdots \cdots \cdots$ Photonengas von $\lambda=6000 \AA$ ).

von Bedeutung sein kann, zeigt z. B. praktisch dieselben Anteile spiegelnd reflektierender Partikel, wie Luft von Stratosphärentemperatur, also überwiegend spiegelnde Reflexion erst bei Wandrauhigkeiten zwischen $10^{-8}$ und $10^{-9} \mathrm{~cm}$.
Dagegen liegen die Verhältnisse für den Elektronenanteil derartiger Plasmen um mehr als eine Zehnerpotenz günstiger, d.h. Elektronengas von $10000^{\circ} \mathrm{K}$ zeigt fast vollständig spiegelnde Reflexion schon bei Wandrauhigkeiten zwischen $10^{-7}$ und $10^{-8} \mathrm{~cm}$.

In stark ionisierenden Feuergasen kann daher der konvektive Wärmeübergang aus der Elektronenkomponente etwas leichter durch glatte Wände beeinflußt werden, als jener aus den schweren Partikeln.

In Abb. 2 ist schließlich auch noch der Bruchteil spiegelnder Reflexion für völlig diffuses Licht einheitlicher Wellenlänge von $\lambda=6000 \AA$ eingetragen, um den Vergleich mit der spiegelnden Reflexion echter Partikel zu erleichtern.

Der kritische Auftreffwinkel ist in diesem Fall $\sin \alpha_{\mathrm{k}}=\lambda / \xi$, die Photonengasdichte $\varrho$, die homogene Geschwindigkeit $c$ gleich der Lichtgeschwindigkeit, die insgesamt in der Zeiteinheit auf die Flächeneinheit fallende Photonenmasse wird nach Gl. (5) $M=\varrho c / 4$ und der Anteil spiegelnd reflektierender Photonen nach Gl. (6) $M_{1}=\varrho c / 4 \cdot(\lambda / \xi)^{2}$, so daß Gl. (9) für homogenes Licht die Form annimmt:

$$
\zeta=M_{1} / M=(\lambda / \xi)^{2} .
$$

Für $\lambda / \xi>1$ wird $\zeta=1$, also alles spiegelnd reflektiert.

Die Luftmoleküle erreichen ähnliche Anteile spiegelnder Reflexion wie die Lichtphotonen daher bei etwa im Verhältnis der Wellenlängen, also bei rund zehntausendfach geringerer Rauhigkeit $\xi$ der Wand.

Die letzte Annäherung des spiegelnden Bruchteiles an eins ist wegen der Auswirkung der Maxwellverteilung der Schwirrgeschwindigkeiten bei echten Partikeln noch etwas schwieriger als bei homogenem, diffusem Licht (Boltzmanngegen Bose-Statistik).

Der Wärmeübergang vom ruhenden Gas an die Wand erfolgt nun nach einem voneinander sehr ver- 
schiedenen Mechanismus bei diffus reflektierenden, und bei spiegelnd reflektierenden Partikeln.

Diffus reflektierende Partikel kommen nach dem Aufprall auf die Wand an dieser zur Ruhe und geben daher zumindest ihre gesamte kinetische Mehrenergie gegenüber der Wand, teilweise auch ihre inneren Energien an die Wand ab und nehmen die Temperatur derselben an.

Zur Berechnung der übertragenen äußeren Energien in der Zeiteinheit auf die Wandflächeneinheit können wir folgendermaßen vorgehen:

Die mit $\mathrm{d} M$ an die Wand gebrachte Energie beträgt:

$$
\begin{aligned}
\mathrm{d} E_{\mathrm{d}}=\frac{\mathrm{d} \varrho}{4 c^{2} \pi} & \int_{\alpha=\operatorname{arc~sin} h / m c \xi}^{\pi / 2} c \sin \alpha \frac{c^{2}}{2} 2 c^{2} \pi \cos \alpha \mathrm{d} \alpha \\
& =\frac{c \mathrm{~d} \varrho}{4}\left[1-(h / m c \xi)^{2}\right] \frac{c^{2}}{2},
\end{aligned}
$$

d. i. also einfach die gesamte kinetische Energie der mit überkritischem Winkel aufprallenden Masse.

Nach Einbeziehung aller $c$ zwischen $h / m \xi$ und unendlich mittels der Gl. (4) folgt:

$$
\begin{aligned}
E_{\mathrm{d}} & =\int_{c=h / m \xi}^{\infty} \frac{c \mathrm{~d} \varrho}{4}\left[1-(h / m c \xi)^{2}\right] \frac{c^{2}}{2} \\
& =\frac{\varrho c_{1}}{2 \sqrt{\pi}} e^{-\left(h / m c_{1}\right) \xi^{2}} \frac{c_{1}{ }^{2}}{2}\left[2+\left(h / m c_{1} \xi\right)^{2}\right] .
\end{aligned}
$$

Mit $h / m c_{1} \xi \rightarrow \infty$ (nur spiegelnde Reflexion) geht $E_{\mathrm{d}}$ gegen null.

Dieser Energiebetrag $E_{\mathrm{d}}$ ist größer, als etwa die thermische Translations-Energie der mit überkritischem Winkel aufprallenden Masse

$$
\frac{\varrho c_{1}}{2 \sqrt{\pi}} e^{-\left(h / m c_{1} \xi\right)^{2}} \frac{3}{4} c_{1}{ }^{2},
$$

worin sich die Tatsache bemerkbar macht, daß die schnelleren Partikel in der Maxwell-Verteilung nicht nur höhere Energie haben, sondern auch häufiger auf die Wand treffen (worauf H. S. Tsien erstmals aufmerksam machte), und kleinere kritische Auftreffwinkel haben, als die langsameren Partikel.

Wenn auch innere Energien in der Höhe von $E_{\mathbf{i}}$ je Masseneinheit ankommenden Gases vorhanden sind, so ist bei völliger Akkomodation der Wärmeübergang aus ihnen offenbar:

$$
E_{\mathrm{d}_{\mathrm{i}}}=\frac{\varrho c_{1}}{2 \sqrt{\pi}} e^{-\left(h / m c_{1} \xi\right)^{2}} E_{\mathrm{i}} .
$$

Für die mit den diffus von der Wand wegfliegenden Partikeln wieder mitgeführte Energie wird meist

${ }^{3}$ E. Sänger, J. Métaux, XXII, 317, 44 [1952]. angenommen, daß sie durch die Wandtemperatur $T_{\mathrm{w}}$ bestimmt wäre zu

$$
E_{\mathrm{r}}=\frac{\varrho c_{1}}{2 \sqrt{\pi}} e^{-\left(h / m c_{1} \xi\right)^{2}} \cdot\left(g c_{\mathrm{v}} T_{\mathrm{w}}\right),
$$

worin $c_{\mathrm{v}}$ die spezifische Wärme des Gases, je nach Akkomodationskoeffizient auch mit Einschluß der inneren Freiheitsgrade ist.

Der Wärmeübergang der spiegelnd reflektierenden Partikel erfolgt nach einem völlig verschiedenen Mechanismus.

Wäre die Wand ein ideal starrer Körper, mit gegenüber der Masse der aufprallenden Partikel unendlich großer Masse, so fände von vornherein überhaupt keine Energieübertragung statt und Reflexions- und Auftreff-Winkel wären streng gleich, ebenso wie Auftreff- und Reflexions-Geschwindigkeit.

Tatsächlich reflektieren die auftreffenden Partikel der Masse $m$ aber an den individuellen Atomen des Kristallgitters der Wand, die die Masse $m_{\mathrm{w}}$ haben mögen.

Während dabei die tangentiale Geschwindigkeitskomponente $(c \cos \alpha)$ des auftreffenden Partikels ungeändert bleibt, wird nach den Gesetzen des elastischen Stoßes die wandnormale Geschwindigkeitskomponente $(c \sin \alpha)$ des auftreffenden Partikels eine Änderung erfahren, die von der Masse $m_{\mathrm{w}}$ und der Geschwindigkeit $c_{\mathrm{w}}$ des getroffenen Wandatoms abhängt ${ }^{3,4}$, und zwar wird die wandnormale Geschwindigkeitskomponente nach dem elastischen Stoß etwa

$$
c \sin a \frac{\left(m / m_{\mathrm{W}}-1\right)-2 c_{\mathrm{w}} / c \sin \alpha}{1+m / m_{\mathrm{W}}}
$$

betragen, auch der Reflexionswinkel wird sich im selben Verhältnis ändern und das Teilchen wird den Energiebetrag

$\frac{m c^{2} \sin ^{2} \alpha}{2} \frac{4\left(m / m_{\mathrm{w}}-c_{\mathrm{w}} / c \sin \alpha\right)}{\left(1+m / m_{\mathrm{w}}\right)^{2}}\left(1+c_{\mathrm{w}} / c \sin \alpha\right)$

an die Gitteratome der Wand übertragen oder von diesen übernehmen. Dieser Betrag hängt somit vom Verhältnis der Atomgewichte der Gaspartikel und der Wandatome ab, und außerdem vom Verhältnis der Schwingungsgeschwindigkeit der Wandatome im Gitter, zur wandnormalen Geschwindigkeitskomponente der Gasteilchen.

Die von $\mathrm{d} M$ in der Zeiteinheit an die Wandflächeneinheit übertragene oder von dieser übernommene Energie beträgt in diesem Fall:

\footnotetext{
${ }^{4}$ E. Sänger, VDI-Forschungsheft 1953, 437.
} 


$$
\begin{aligned}
\mathrm{d} E_{\mathrm{s}} & =\frac{\mathrm{d} \varrho}{4 c^{2} \pi} \int_{\alpha=0}^{\operatorname{arc} \sin h / m c \xi} c \sin \alpha \frac{c^{2} \sin ^{2} \alpha}{2} \frac{4\left(m / m_{\mathrm{W}}-c_{\mathrm{W}} / c \sin \alpha\right.}{\left(1+m / m_{\mathrm{W}}\right)^{2}}\left(1+c_{\mathrm{w}} / c \sin \alpha\right) 2 c^{2} \pi \cos \alpha \mathrm{d} \alpha \\
& =\frac{4 m / m_{\mathrm{W}}}{\left(1+m / m_{\mathrm{W}}\right)^{2}} \frac{c \mathrm{~d} \varrho}{4} \frac{c^{2}}{2}\left[\frac{1}{2}(h / m c \xi)^{4}+\frac{2}{3} \frac{c_{\mathrm{W}}}{c}\left(1-\frac{m_{\mathrm{W}}}{m}\right)(h / m c \xi)^{3}-\frac{c_{\mathrm{w}}^{2}}{c^{2}} \frac{m_{\mathrm{W}}}{m}(h / m c \xi)^{2}\right],
\end{aligned}
$$

soweit $h / m c \xi<1$, andernfalls wird

$$
\mathrm{d} E_{\mathrm{s}}=\frac{4 m / m_{\mathrm{W}}}{\left(1+m / m_{\mathrm{w}}\right)^{2}} \frac{c \mathrm{~d} \varrho}{4} \frac{c^{2}}{2}\left[\frac{1}{2}+\frac{2}{3} \frac{c_{\mathrm{w}}}{c}\left(1-\frac{m_{\mathrm{W}}}{m}\right)-\frac{c_{\mathrm{w}}^{2}}{c^{2}} \frac{m_{\mathrm{W}}}{m}\right] .
$$

Nach Einbeziehung aller $c$ zwischen null und unendlich mittels Gl. (4) folgt:

$$
\begin{gathered}
E_{\mathrm{s}}=\int_{c=0}^{h / m \xi} \frac{4 m / m_{\mathrm{W}}}{\left(1+m / m_{\mathrm{W}}\right)^{2}} \frac{c \mathrm{~d} \varrho}{4} \frac{c^{2}}{2}\left[\frac{1}{2}+\frac{2}{3} \frac{c_{\mathrm{W}}}{c}\left(1-\frac{m_{\mathrm{W}}}{m}\right)-\frac{c_{\mathrm{W}}^{2}}{c^{2}} \frac{m_{\mathrm{W}}}{m}\right]+\int_{c=h / m \xi}^{\infty} \frac{4 m / m_{\mathrm{W}}}{\left(1+m / m_{\mathrm{W}}\right)^{2}} \frac{c \mathrm{~d} \varrho}{4} \frac{c^{2}}{2}\left[\frac{1}{2}(h / m c \xi)^{4}\right. \\
\left.+\frac{2}{3} \frac{c_{\mathrm{W}}}{c}\left(1-\frac{m_{\mathrm{W}}}{m}\right)(h / m c \xi)^{3}-\frac{c_{\mathrm{m}}^{2}}{c^{2}} \frac{m_{\mathrm{W}}}{m}(h / m c \xi)^{2}\right]=\frac{4 m / m_{\mathrm{W}}}{\left(1+m / m_{\mathrm{W}}\right)^{2}} \frac{\varrho c_{1}}{2 \sqrt{\pi}} \cdot \frac{c_{1}{ }^{2}}{2}\left\{1-e^{-\left(h / m c_{1} \xi\right)^{2}}\left[1+\left(h / m c_{1} \xi\right)^{2}\right]\right. \\
\left.\quad+\frac{c_{\mathrm{W}}}{c_{1}}\left(1-\frac{m_{\mathrm{W}}}{m}\right)\left[\int_{0}^{h / m c_{1} \xi} e^{-x^{2}} \mathrm{~d} x-e^{-\left(h / m c_{1} \xi\right)^{2}}\left(h / m c_{1} \xi\right)\right]+\frac{c_{\mathrm{W}}^{2}}{c_{1}{ }^{2}} \frac{m_{\mathrm{W}}}{m}\left[e^{-\left(h / m c_{1} \xi\right)^{2}}-1\right]\right\}
\end{gathered}
$$

Für $c_{\mathrm{w}}=0$ (ruhende Gitteratome) und $h / m c \xi_{1}=\infty$ (völlig glatte Wand) wird die geschwungene Klammer gleich eins, es folgt der bekannte optische Energieaustausch. Für $h / m c_{1} \xi=0$ (völlig rauhe Wand) verschwindet der elastische Energieaustausch.

Dieser Wärmeübergangsbetrag kann je nach den vorliegenden Verhältnissen positiv (Wärme vom Gas zur Wand) oder negativ (umgekehrt) sein.

Tatsächlich wird in vielen Fällen die Wandtemperatur $T_{\mathrm{w}}=m_{\mathrm{w}} c_{\mathrm{w}}^{2} / 3 k$ gegenüber der Gastemperatur $T=m c_{\mathrm{m}}^{2} / 3 k=m c_{1}^{2} / 2 k$ keineswegs vernachlässigbar sein, an den Außenoberflächen von Überschallflugzeugen wird sie sogar oft erheblich höher als die Temperatur der ungestörten Luft sein, so daß tatsächlich Energie von den Wandatomen an die spiegelnd reflektierenden Luftmoleküle übertragen wird, diese also kühlend auf die Wand wirken.

Aus Gl. (17) folgt auch ohne weiteres, daß der Energieaustausch zwischen Wand und spiegelnd reflektierendem Gas nicht etwa verschwindet, wenn die Temperaturen $T_{\mathrm{w}}$ und $T$ gleich geworden sind, ebensowenig, wie beim Strahlungsaustausch zwischen einem strahlenden Gas und einer Wand. Durch Nullsetzen der geschwungenen Klammer in Gl. (17) folgt vielmehr die Bedingung, unter der der Energieaustausch im Mittel bei spiegelnder Reflexion verschwindet, also auch Einfalls- und Reflexionswinkel im Mittel untereinander gleich sind. Der individuelle Energieaustausch der Einzelpartikel verschwindet nach den Gesetzen elastischen Stoßes bekanntlich, wenn die Partikelgeschwindig- keiten sich verhalten wie $c_{\mathrm{w}} / c=m / m_{\mathrm{w}}$, infolge der Maxwell-Verteilung der $c$ im Gas gibt es bei einem vorgegebenen Zustand neben solchen Teilchen, die keinen Energieaustausch haben, immer auch andere, die Energie an die Wand abgeben, und wieder andere, die Energie von der Wand übernehmen. Die durch Nullsetzen der Klammer in Gl. (17) erhaltene Beziehung definiert daher jenen mittleren Gesamtzustand, wo sich die Wärmeabgabe an die Wand mit der Wärmeaufnahme aus ihr gerade ausgleichen. Dieser Zustand hängt nicht nur von $\mathrm{m} / \mathrm{m}_{\mathrm{w}}$ ab, sondern auch von $h / m c_{1} \xi$, weil die Geschwindigkeitsbereiche spiegelnd reflektierender Teilchen in der Maxwell-Verteilung sich mit ihm ändern.

Wir können die drei Komponenten $E_{\mathrm{d}}, E_{\mathrm{r}}$ und $E_{\mathrm{s}}$ des konvektiven Wärmeüberganges auf das sogenannte Konvektionsprodukt $K=\gamma H_{0} c_{\mathrm{m}}$ (aus spezifischem Gasgewicht $\gamma$, Enthalpie $H_{0}$ des ruhenden Gases und mittlerer Teilchengeschwindigkeit $\left.c_{\mathrm{m}}\right)^{5}$ beziehen und dieses Verhältnis als dimensionslosen Beiwert des Wärmeüberganges benützen.

Für die äußeren Freiheitsgrade allein wird das Konvektionsprodukt

$$
K=\frac{5}{6} \varrho c_{\mathrm{m}}^{3}=\frac{5}{6}\left(\frac{3}{2}\right)^{3 / 2} \varrho c_{1}{ }^{3} .
$$

Damit folgt der Beiwert des Wärmeüberganges aus dem Aufprall des diffus reflektierenden Massenanteiles aus Gl. (11) und (18):

${ }^{5}$ I. Sänger-Bredt, Z. Naturforschg. 8a, 12, 796 [1953]. 


$$
E_{\mathrm{d}} / K=\frac{1}{5} \sqrt{\frac{2}{3 \pi}} e^{-\left(h / m c_{1} \xi\right)^{2}}\left[2+\left(h / m c_{1} \xi\right)^{2}\right]
$$

und aus dem Rückprall desselben Massenanteiles nach Gl. (13) und (18)

$$
E_{\mathrm{r}} / K=-\frac{1}{5} \sqrt{\frac{2}{3 \pi}} e^{-\left(h / m c_{1} \xi\right)^{2}} \frac{m_{\mathrm{W}}}{m}\left(\frac{c_{\mathrm{W}}}{c_{1}}\right)^{2}
$$

und schließlich der Beiwert des Wärmeüberganges aus dem spiegelnd reflektierenden Massenanteil nach Gl. (17) und (18)

$$
\begin{aligned}
& E_{\mathrm{s}} / K=\frac{1}{5} \sqrt{\frac{2}{3 \pi}} \frac{4 m / m_{\mathrm{w}}}{\left(1+m / m_{\mathrm{w}}\right)^{2}} \cdot\left\{1-e^{-\left(h / m c_{1} \xi\right)^{2}}\left[1-\left(h / m c_{1} \xi\right)^{2}\right]\right. \\
&+\frac{c_{\mathrm{w}}}{c_{1}}\left(1-\frac{m_{\mathrm{w}}}{m}\right)\left[\int_{0}^{h / m c_{1} \xi} e^{-x^{2}} \mathrm{~d} x-e^{-\left(h / m c_{1} \xi\right)^{2}}\left(h / m c_{1} \xi\right)\right] \\
&\left.+\frac{c_{\mathrm{w}}^{2}}{c_{1}{ }^{2}} \frac{m_{\mathrm{w}}}{m}\left[e^{-\left(h / m c_{1} \xi\right)^{2}}-1\right]\right\} .
\end{aligned}
$$

Während also der erste dieser drei Wärmeübergangsbeiwerte nur vom kritischen Wert $h / m c_{1} \xi$ abhängt, spielen bei den beiden andern auch die Verhältnisse der Atomgewichte von Gaspartikeln und Wandatomen und von deren Temperaturen ihre Rolle.

\section{Strömendes Gas}

Strömendes Gas kann in unmittelbarer Nähe einer festen Wand bekanntlich eine wandparallele Strömungsgeschwindigkeit nur dann besitzen, wenn es keine Strömungsgrenzschicht bildet, also z. B. wenn die freie Weglänge der Gasteilchen groß ist gegenüber der Länge der beströmten Wand (z. B. sehr große Flughöhe), so daß jedes überhaupt mit der Wand kollidierende Teilchen nur einmal auf die Wand trifft, oder aber näherungsweise auch noch, wenn die Wandrauhigkeit $\xi$ so klein ist, daß die Zahl der diffus reflektierenden Partikel vernachlässigbar wird.

Nur unter diesen Voraussetzungen darf die in den folgenden Überlegungen zum strömenden Gas benützte wandnahe Strömungsgeschwindigkeit $v$ gleich der Fluggeschwindigkeit bzw. der Geschwindigkeit in der freien Strömung gesetzt werden.

Führen wir zu den Größen der Abb. 1 also noch die Strömungsgeschwindigkeit $v$ des Gases in Wandnähe ein, d. h. die geordnete, wandparallele Systemgeschwindigkeit aller ankommenden Gaspartikel, so entstehen die in Abb. 3 dargestellten Verhältnisse. Die Geschwindigkeitsresultierende aus System- und Schwirr-Geschwindigkeit wird

$$
w^{2}=v^{2}+c^{2}+2 v c \cos \alpha \cos \beta
$$

und der Neigungswinkel $\alpha_{c}$ derselben gegen die Begrenzungswand

$\sin \alpha_{\mathrm{c}}=\frac{c \sin \alpha}{w}=\frac{\sin \alpha}{\sqrt{1+v^{2} / c^{2}+2 v / c \cos \alpha \cos \beta}}$.

Der Neigungswinkel $\alpha_{c}$ wird also gleich dem Grenzauftreffwinkel $\alpha_{k}$, sobald die wandnormale Geschwindigkeitskomponente der Partikel $(c \sin \alpha)$ gleich ist $h / m \xi$, genau wie beim ruhenden Gas.

Die wandparallele Strömungsgeschwindigkeit $v$ ist daher ohne jeden Einfluß auf die Tatsache, ob ein bestimmtes Teilchen spiegelnd oder diffus reflektiert wird, diese Tatsache wird nur durch die von der thermischen Schwirrgeschwindigkeit herrührende, wandnormale Geschwindigkeitskomponente entschieden, und zwar, weil sich mit wachsender Auftreffgeschwindigkeit w sowohl der wirkliche, als auch der kritische Auftreffwinkel nach den Gln. (1) und (22) in ganz gleicher Weise ändern.

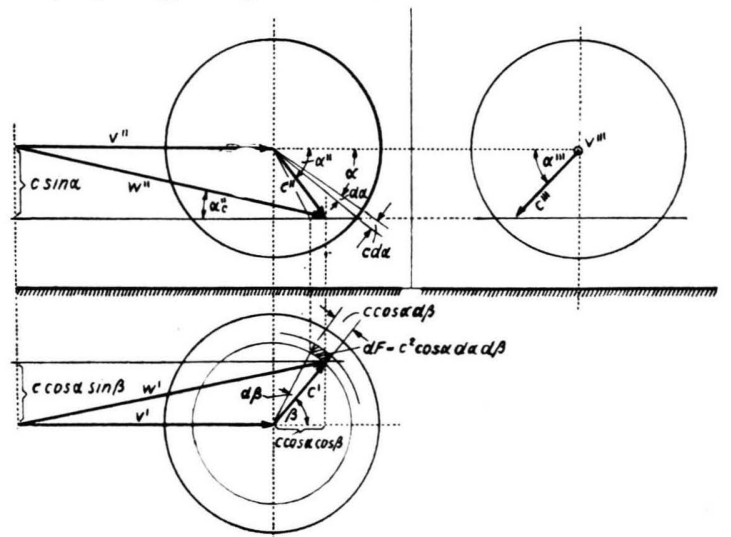

Abb. 3. Geometrie des Aufpralles der Partikel strömenden Gases auf eine Wand.

Da bei plattenparalleler Strömung bekanntlich (l. c. ${ }^{1,2}$ ) auch die Zahl der sekundlich auf die Flächeneinheit der Platte prallenden Teilchen unabhängig von der Systemgeschwindigkeit $v$ gleich dem durch Gl. (7) gegebenen Wert $\varrho c_{1} / 2 \sqrt{\pi}$ ruhenden Gases bleibt, gilt dasselbe auch für den Bruchteil $\zeta$ spiegelnd reflektierender Teilchen, d.h. Gln. (8) und (9) und damit Abb. 2 gelten nicht nur für ruhendes, sondern auch für strömendes Gas, wie man auch durch Einzelausrechnung der Verhältnisse der Abb. 3 analog zu jenen der Abb. 1 leicht zeigen kann.

Unterschiede zwischen strömendem und ruhendem Gas entstehen erst bei der Bestimmung der tatsächlichen Reibungskräfte und Wärmeübergänge. 
Die spiegelnd reflektierenden Partikel liefern naturgemä $ß$ keine Beiträge zur Reibung, wohl aber zum Wärmeübergang.

Der wandparallele Impuls der sekundlich von der Flächeneinheit diffus reflektierenden.Partikel vor ihrem Auftreffen betrug offenbar

$$
\tau=\frac{v c_{1} \varrho}{2 \sqrt{\pi}} e^{-\left(h / m c_{1} \xi\right)^{2}} .
$$

Dieser Impuls je Zeiteinheit ist gleich der Reibungskraft je Flächeneinheit, die also linear mit der Systemgeschwindigkeit $v$ anwächst. Der Reibungsbeiwert in der üblichen Definition wird

$\tau / q=\frac{c_{1}}{v} \frac{1}{\sqrt{\pi}} e^{-\left(h / m c_{1} \xi\right)^{2}}=\frac{a}{v} \sqrt{\frac{2}{\varkappa \pi}} e^{-\left(h / m c_{1} \xi\right)^{2}}$,

wenn man statt der wahrscheinlichsten Partikelgeschwindigkeit $c_{1}{ }^{2}=2 g R T$ noch die Schallgeschwindigkeit $a^{2}=\varkappa g R T$ im Gas einführt.

Der Wärmeübergang infolge diffus reflektierender Teilchen ist wegen seiner quadratischen Ab- hängigkeit von der resultierenden Aufprallgeschwindigkeit $w$ etwas umständlicher zu ermitteln.

Wir beschränken uns wieder auf die mit den haftenden Teilchen an die Wand gebrachte Energie, während die von ihnen bei der diffusen Reflexion wieder mitgenommene Energie bekanntlich dadurch bestimmt sein soll, daß die haftenden und dann diffus reflektierenden Teilchen während der durchaus endlichen Verweilzeiten an der Wand deren Temperaturen annehmen, so daß dafür Gl. (13) gilt.

Wir greifen in Abb. 3 zunächst wieder den Massenanteil $\mathrm{d} \varrho$ fast homogener Schwirrgeschwindigkeit $c$ heraus. Beachten wir, $\operatorname{da} \beta \operatorname{d} \varrho \mathrm{d} F / 4 c^{2} \pi$ hier die durch das Oberflächenelement $\mathrm{d} F=c^{2} \cos \alpha \mathrm{d} \alpha \mathrm{d} \beta$ der gezeichneten Geschwindigkeitskugel gehende Teilchenmasse ist, und daß deren kinetische Energie $\mathrm{d} \varrho w^{2} / 2$ beträgt, so wird die mit überkritischem Aufprallwinkel an die Wand gebrachte Energie

$$
\mathrm{d} E_{\mathrm{d}}=\frac{\mathrm{d} \varrho}{4 c^{2} \pi} \int_{\alpha=\operatorname{arc} \sin h / m c \xi}^{\pi / 2} \int_{\beta=0}^{2 \pi} c \sin \alpha \frac{v^{2}+c^{2}+2 v c \cos \alpha \cos \beta}{2} c^{2} \cos \alpha \mathrm{d} \alpha \mathrm{d} \beta=\frac{c \mathrm{~d} \varrho}{4}\left[1-(h / m c \xi)^{2}\right]\left(\frac{v^{2}+c^{2}}{2}\right),
$$

d. i. einfach wieder die gesamte kinetische Energie der mit überkritischem Winkel aufprallenden Masse.

Bezieht man mit Hilfe der Gl. (4) nun wieder alle Partikel mit den verschiedenen, zu diffuser Reflexion führenden Geschwindigkeiten $c$ zwischen $h / m \xi$ und unendlich in die Rechnung ein, so wird die gesamte, auf die Wand übertragene Energie:

$$
\begin{aligned}
& E_{\mathrm{d}}=\int_{c=h / m \xi}^{\infty} \frac{c \mathrm{~d} \varrho}{4}\left[1-\left(h / m c_{1} \xi\right)^{2}\right]\left(\frac{v^{2}+c^{2}}{2}\right) \\
& =\frac{\varrho c_{1}}{2 \sqrt{\pi}} e^{-\left(h / m c_{1}\right)^{2} \xi}\left\{\frac{v^{2}}{2}+\frac{c_{1}^{2}}{2}\left[2+\left(h / m c_{1} \xi\right)^{2}\right]\right\} .
\end{aligned}
$$

Für $h / m c_{1} \xi \rightarrow \infty$ geht $E_{\mathrm{d}}$ naturgemäß gegen null, jedoch wesentlich langsamer als $\tau$.

Hier macht sich auch wieder in der geschwungenen Klammer die Tatsache bemerkbar, daß die schnellen Partikel in der Maxwell-Verteilung nicht nur höhere Energie haben, sondern auch häufiger auf die Wand treffen und kleinere kritische Auftreffwinkel $\alpha_{\mathrm{k}}$ haben, als die energieärmeren.

Falls die Gasmoleküle außer der hier betrachteten kinetischen Energie auch noch innere Energien mit sich führen und an die Wand übertragen, etwa Rotations-, Schwingungs-, Termanregungs-, Dissoziations- oder Ionisations-Energien, so sind diese in der geschwungenen Klammer als weitere Summanden anzufügen.

Den Wärmeübergangsanteil der spiegelnd reflektierenden Partikel kann man in analoger Weise wie bei den Gln. (16) und (17) ruhenden Gases ermitteln. Da die Systemgeschwindigkeit $v$ keinen Einfluß auf die wandnormalen Geschwindigkeitskomponenten und damit auf die kritischen Auftreffwinkel hat, sind die beiden genannten Gleichungen hier unmittelbar zu verwenden.

Das Konvektionsprodukt $K=\gamma H_{0} c_{\mathrm{m}}$ wird für die äußeren Energien allein nunmehr

$K=\frac{\varrho c_{m}}{2}\left(\frac{5}{3} c_{\mathrm{m}}^{2}+v^{2}\right)=\sqrt{\frac{3}{2}} \frac{\varrho c_{1}}{2}\left(\frac{5}{2} c_{1}{ }^{2}+v^{2}\right)$

und damit der Beiwert des Wärmeüberganges aus dem Aufprall des diffus reflektierenden Massenanteiles aus Gl. (26) und (27):

$E_{\mathrm{d}} / K=\frac{1}{\sqrt{6 \pi}} e^{-\left(h / m c_{1} \xi\right)^{2}} \frac{v^{2}+c_{1}{ }^{2}\left[2+\left(h / m c_{1} \xi\right)^{2}\right]}{v^{2}+{ }^{5} / 2 c_{1}{ }^{2}}$

und aus dem Rückprall desselben Massenanteiles nach Gl. (13) und (27):

$E_{\mathbf{r}} / K=-\frac{1}{\sqrt{6 \pi}} e^{-\left(h / m c_{1} \xi\right)^{2}} \frac{m_{\mathrm{W}}}{m} \frac{c_{\mathrm{W}}^{2}}{v^{2}+{ }^{5} / 2 c_{1}{ }^{2}}$

und schließlich der Beiwert des Wärmeüberganges aus dem spiegelnd reflektierenden Massenanteil nach Gl. (17) und (27): 


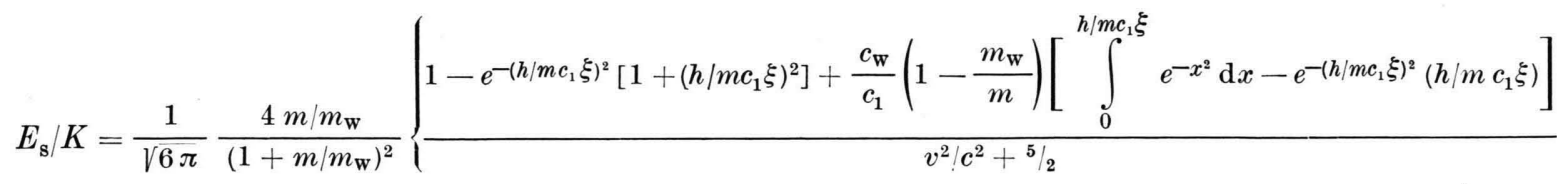

$$
\begin{aligned}
& \left.+\frac{\frac{c^{2} \mathrm{w}}{c_{1}{ }^{2}} \frac{m_{\mathrm{W}}}{m}\left[e^{-\left(h / m c_{1} \xi\right)^{2}}-1\right]}{v^{2} / c^{2}+{ }^{5} / 2}\right\} .
\end{aligned}
$$

Mit $v=0$ gehen diese drei Gleichungen natürlich in die entsprechenden Gl. (19), (20) und (21) über.

Während der erste Wärmeübergangsbeiwert mit anwachsender Strömungsgeschwindigkeit asymptotisch dem konstanten Maximalwert

$$
\left(E_{\mathrm{d}} / K\right)_{v \rightarrow \infty}=\frac{1}{\sqrt{6 \pi}} e^{-\left(h / m c_{1} \xi\right)^{2}}
$$

zustrebt, gehen die beiden andern Beiwerte unter gleichen Bedingungen gegen null.

Man kann indes bei strömenden Gasen den Wärmeübergang statt auf das Konvektionsprodukt auch auf die Reibungsleistung $\tau v$ beziehen und erhält beispielsweise mit Gl. (26) und (23):

$$
E_{\mathrm{d}} / \tau v=\frac{1}{2}+\frac{1}{\varkappa} \frac{a^{2}}{v^{2}}\left[2+\left(h / m c_{1} \xi\right)^{2}\right] .
$$

Beim diffusen Wärmeübergang tritt hier zu dem elementaren Beiwert

$$
\mathrm{d} E_{\mathrm{d}} / \tau v=\frac{m v^{2} / 2}{m v^{2}}=\frac{1}{2}
$$

plastischen Aufpralles von Teilchen ohne Wärmebewegung noch ein Beitrag aus der Wärmebewegung, der z. B. zur Folge hat, daß ein ruhendes Gas mit $v=0$ durchaus endlich großen Wärmeübergang bewirkt.

Aus Gl. (13) und (23) folgt

$$
E_{\mathrm{r}} / \tau v=g c_{\mathrm{v}} T_{\mathrm{w}} / v^{2}
$$

und schließlich aus Gl. (17) und (23)

$$
E_{\mathrm{s}} / \tau v=\frac{1}{x} \frac{a^{2}}{v^{2}} \frac{4 m / m_{\mathrm{W}}}{\left(1+m / m_{\mathrm{W}}\right)^{2}}\left\{\frac{1-e^{-\left(h / m c_{1} \xi\right)^{2}\left[1+\left(h / m c_{1} \xi\right)^{2}\right]+\frac{c_{\mathrm{W}}}{c_{1}}\left(1-\frac{m_{\mathrm{W}}}{m}\right)\left[\int_{0}^{h / m c_{1} \xi} e^{-x^{2}} \mathrm{~d} x-e^{-\left(h / m c_{1} \xi\right)^{2}}\left(h / m c_{1} \xi\right)\right]}}{e^{-\left(h / m c_{1} \xi^{2}\right)}}-\frac{\frac{c^{2} \mathrm{w}}{c_{1}{ }^{2}} \frac{m_{\mathrm{W}}}{m}\left[e^{-\left(h / m c_{1} \xi\right)^{2}}-1\right]}{\left.e^{-\left(h / m c_{1} \xi\right)^{2}}\right\} \cdot(33)}\right.
$$

Während der erste Wärmeübergangsbeiwert bei $v=0$ mit unendlich beginnend, mit wachsender Strömungsgeschwindigkeit asymptotisch gegen den
Grenzwert $E_{\mathrm{d}} / \tau v=1 / 2$ verläuft, gehen die beiden andern Beiwerte unter gleichen Bedingungen wieder gegen null. 